\title{
Supporting the Knowledge Life-Cycle
}

\author{
Luciënne Blessing and Ken Wallace \\ Engineering Design Centre, University of Cambridge, UK
}

Key words: Knowledge life-cycle, Product development, Design process

\begin{abstract}
This paper describes the mismatch between the information sources available to designers and the information sources accessed by designers. Possible reasons for this mismatch are accessibility, availability and trustworthiness. Current support for product development does not deal with this issue because only part of the knowledge life-cycle is addressed. A model of the knowledge life-cycle is introduced that focuses on knowledge generation. To support the knowledge life-cycle, a system is introduced based on a model of the design process. This system, PROSUS, is discussed and the issues needing further development identified.
\end{abstract}

\section{INTRODUCTION}

Engineering design is a knowledge-intensive activity. The knowledge used comes from a variety of sources, including those within the company as well as those outside, and those related to work as well as those not related to work. This knowledge includes that gained from positive and negative experiences. Knowledge is stored as information and data in handbooks, drawings, documents, electronic means and, in particular, in the heads of individuals. As products become more complex and competition intensifies, it is essential to make the maximum use of the available knowledge and to deliver that knowledge in the appropriate form at the right time in the product development process. Developing a knowledge-intensive system to support this is a challenging task because of the following knowledge-related characteristics of engineering design:

- wide variety of issues to be addressed; 
- complex relationships between issues;

- dynamic nature of the issues.

These characteristics make the development of knowledge-intensive CAD a challenging task and are the reason why the design process will continue to be driven by human designers.

\section{$1.1 \quad$ Wide variety}

Knowledge of various technical and non-technical domains has to be combined when designing engineering products in order to address the multitude of life-cycle issues, and also the requirements and constraints of all business functions and external parties. This variety is expressed, or should be expressed, in the requirements specification. Several methods and models have been developed to help designers obtain an overview of the relevant issues. Examples include: checklists for formulating requirements, e.g. [Pahl and Beitz 1996]; QFD to assist designers with the translation of customer needs into technical demands [Clausing 1994]; Pugh's Information Input model [Pugh 1991]; and the overview of properties of technical systems and life-cycle demands from the environment as given by [Hubka and Eder 1988].

\section{$1.2 \quad$ Complex relationships}

Engineering design is knowledge intensive because of the wide variety of issues involved and because of the complex relationships between the issues. Hubka and Eder illustrate this by describing the multitude of relationships between design properties. According to Altschuller [1984] a design problem exists because of a contradiction between the requirements placed on two related properties, e.g. high stiffness and low weight.

The existence of these relationships requires the ability to make tradeoffs. Some argue that design is essentially a process of trading off requirements and constraints. Every design project is different because at least some of the requirements or constraints have changed. This means that there is no unique route to a solution and no single solution to a design problem.

\subsection{Dynamic nature}

Engineering design is also knowledge intensive because of the dynamic nature of the knowledge applied. This is captured in the term experience: every step in a design project adds knowledge, at least to the individual 
involved. Design is a continuous learning process: the knowledge base changes continuously. For systems to be truly knowledge intensive, it is necessary for them to take into account the dynamic nature of the knowledge. This knowledge can relate to the product being designed as well as to the design process being undertaken. Currently most of the knowledge captured explicitly relates to the product.

\section{$1.4 \quad$ Focus}

Our work focuses on capturing the knowledge that is being created and used in a project, as and when it is being created and used. In this way the variety of knowledge and the "trade-off" knowledge is captured most comprehensively, whilst doing justice to its dynamic nature.

The next section discusses why capturing the knowledge that is being generated and used, rather than simply the product-related outputs of the design process, is the focus of our work. Section 3 introduces the knowledge life-cycle and provides an outline of the requirements of a support tool based on this life-cycle model. Section 4 gives a summary of the PROSUS system, which addresses the life-cycle issues we consider essential. Section 5 discusses PROSUS in the light of the knowledge life-cycle. The concluding section presents the issues that still need to be addressed.

\section{KNOWLEDGE USE}

The need to capture knowledge as it is being generated and used suggests that the knowledge currently captured in various forms is not sufficient. The wish of many companies to store more knowledge seems strange at a time when more knowledge and information than ever are available. However, comparing some research findings shows an interesting mismatch between what is available and what is being used.

\subsection{Mismatch between availability and use}

University libraries, such as those in Cambridge, need about 4 kilometres of shelving space to stock the most essential scientific literature generated in one year. About 1.5 million new books are published in the world each year [Ehrlenspiel 1997]. The amount of information stored within a company has also reached enormous proportions. For example, the development of an aero engine produces approximately 40,000 documents. At Rolls-Royce the technical library is reported to support 60 million sheets of A4 
documentation per quarter [Marsh 1997]. New technologies are increasingly used to reduce storage space and increase availability. The large amount of shelving space needed for books and documents can be reduced to a few metres when CD ROMs are used as the storage medium. In 1997, around 200,000 new pages were installed on the Internet each day and the number is growing rapidly [Ehrlenspiel 1997]. Some are convinced that one day we will never need to visit a library because we will simply search the WWW and download the relevant information directly into our computers.

At the same time, observations in industry indicate that the single greatest factor (30\% of cases) underlying wrong decisions is lack of information, rather than lack of experience [Frankenberger and Badke-Schaub 1997]. This is not due to the time researchers spent searching for information. Several researchers found the proportion of a designer's time absorbed by information acquisition activities to be between 20-30\% [Court 1995, Marsh 1997]. Marsh also found that the majority of information is obtained from personal contacts, confirming earlier findings such as [Lera et al 1984, Hales 1987, Blessing 1994]. In $78 \%$ of cases these contacts retrieved the information from their memories. Formally recorded information was obtained on average twice each day, but was accessed directly in only $55 \%$ of cases observed, that is it is often obtained through others. This is interesting because the company studied by Marsh stores large amounts of information about past cases. It is also interesting to note that despite new technologies that should make retrieval easier and quicker, the percentages do not seem to have changed significantly.

The effect on the working environment of using personal contacts for obtaining information is large. Marsh [Marsh 1997] observed that there is less than a $50 \%$ chance of working continuously for longer than 8 minutes before an interruption occurs stemming from a designer's own requirements for information or those of colleagues. The chance of working for 30 minutes or more is less than $25 \%$. Also, as most searches for information involve two people, the required effort could be reduced by up to $50 \%$ if a good non-human source of information was available.

On the one hand the findings suggest superabundant information, on the other hand a strong, almost sole, reliance on personal contacts. Three possible reasons are:

- accessibility of documented information;

- availability of relevant information;

- trustworthiness of information.

Supporting evidence for all three reasons is presented below. 


\subsection{Accessibility}

Several studies have concluded that it is often difficult to find the relevant information. Earlier studies, summarised in [Lera et al 1984], have revealed that information was perceived as either inaccessible, unavailable or not in an applicable form, and that consulting written information was seen as time consuming. The latter perception was also observed in more recent experimental studies where the subjects relied on the experiment leader to answer questions. This was presumably because of the ease with which the information could be obtained, even though all the information was available to them in written form [Kuffner and Ullman 1991]. These findings were confirmed in the experiments described in [Blessing 1994] and [Klaubert 1998]. The studies discussed earlier in this section show similar results by indicating the large number of personal contacts used as information sources, despite the fact that more and more information is available in electronic format intended to accelerate access.

One of the problems appears to be that sources such as the Internet have grown so large and diverse that it is not unusual for a search to result in several thousand "hits", of which only a few are relevant. Commercial claims (KPMG consultants in [Marsh 1997]) are that 95\% of filed documents are only ever retrieved once, and that is to discard them. A major problem of accessing existing databases is because their indexing systems were set up before knowing what information will eventually be requested. Research is ongoing into making large legacy databases containing product and process data more accessible, without resorting to keywords and predefined indices [Carlton 1998].

\subsubsection{Availability}

Relevant information is not always available. Marsh looked into the different types of information and their impact on the working time of designers. He showed that on average $24 \%$ of each designer's working day was spent obtaining or providing information. This $24 \%$ was made up as follows:

$-6 \%$ of time was spent on product-based information acquisition. This is anything relating directly to factual knowledge about the product, its description, specification, and associated design data.

- $11 \%$ of time was spent on process-based information. This information represents knowledge about how to perform tasks, approach problems, etc.

$-3 \%$ of time was spent on resource information. This includes knowledge about how to operate complex computer-based tools, the information provided by standards, formal representation of "know-how", etc. 
$-4 \%$ of time was spent on miscellaneous information. This information is concerned with arranging meetings, organising information flow, etc.

In particular, process-based information was difficult to find in a generally accessible form. Product-based information was only partially available. The lessons learnt, the rationale behind the product, and the various alternatives that were considered but rejected, were normally only to be found in designers' notebooks, if at all. This, in particular, is the knowledge that builds up into experience: the information that makes designers "experts".

The knowledge of experts is considered difficult to capture. In addition, this knowledge can be contradictory, as research in the field of knowledge acquisition has clearly demonstrated. Marsh [Marsh 1997] concluded from his study that in the engineering environment he observed only a quarter of queries relating to design expertise, answered with reference to past experience, required the abilities of an expert to evaluate, interpret and explain. The single greatest use made of experts was to provide an awareness of alternatives, of concerns, of work carried out in the past, of problem symptoms, etc. It is significant that the contributions made by experts in the majority of cases was comparable to the type of support that could be provided by structured information management and knowledge capture, rather than the replication of human cognitive abilities.

The need for this knowledge to be more widely available has led to companies applying a variety of approaches to capture expertise. RollsRoyce, for example, has started a "lessons learnt" database and is engaged in a project to capture knowledge from experts using novices to undertake the knowledge acquisition. British Gas, on the other hand, is employing knowledge engineers to capture the knowledge. The techniques used have one significant disadvantage, namely the retrospective nature of the resulting information. This issue is discussed in section 3 .

\subsection{Trustworthiness}

Trust in the information supplied is an important reason why information sources, even those generated within a company, may not be accessed and why designers prefer to go to experts who are considered to be more knowledgeable and up-to-date. This factor should not be underestimated. Adding to a database the source, date of entry and, most importantly, the context in which the information was used, could help built trust and confidence. 


\section{KNOWLEDGE LIFE-CYCLE}

The previous section suggests that much information is available, but what is requested most, namely expertise, is not available or difficult to access. As this is the knowledge generated during the design activity, knowledge-intensive $\mathrm{CAD}$ represents a challenging task.

A knowledge-intensive environment cannot rely on a knowledge usecycle, that is Capture-Store-Retrieve-Reuse. Reuse is too limited because it suggests that the knowledge being used does not change. Our use of the word knowledge includes within it the knowledge of where and when it can be applied. As such, knowledge includes the expertise that is needed to determine relevancy and build trust. Using this concept of knowledge means that every time knowledge is used new knowledge is generated, even if it is only further detailed knowledge about how the knowledge was applied.

When developing any knowledge-based support for design it is important that the whole knowledge life-cycle is taken into account, see Figure 1. In order to know what to capture, it is important to investigate what is used. What is captured needs to be stored in such a way that it is easy to retrieve and is relevant to the situation in which it is going to be used. Use of knowledge may be successful, if, when it is retrieved and possibly adapted, it is useful in the new situation. Use of knowledge may also be unsuccessful, if when it is retrieved it is not useful. However, in both cases new knowledge is generated. This new knowledge may replace or refine old knowledge, or be a new addition. The knowledge base needs to be continually updated. This is like a basic learning process. A more sophisticated learning process involves generating meta-knowledge (knowledge about knowledge) or generic knowledge from what has been captured and stored. The dynamic nature of knowledge is determined by the generation of new knowledge as a result of knowledge use, that is a continual "learning process".

Figure 1 can be seen as the representation of the knowledge life-cycle of an individual, of a team, and of a company. In each of these cases, the term "outside sources" takes on a different meaning. The outside sources themselves have their own knowledge life-cycles. Knowledge from outside sources can be captured directly, for example at the start of building a knowledge base, or it can be captured indirectly, for example when certain knowledge elements are being used and are captured as a consequence of this use. The latter is the preferred route as the relevance to work in hand is guaranteed. 


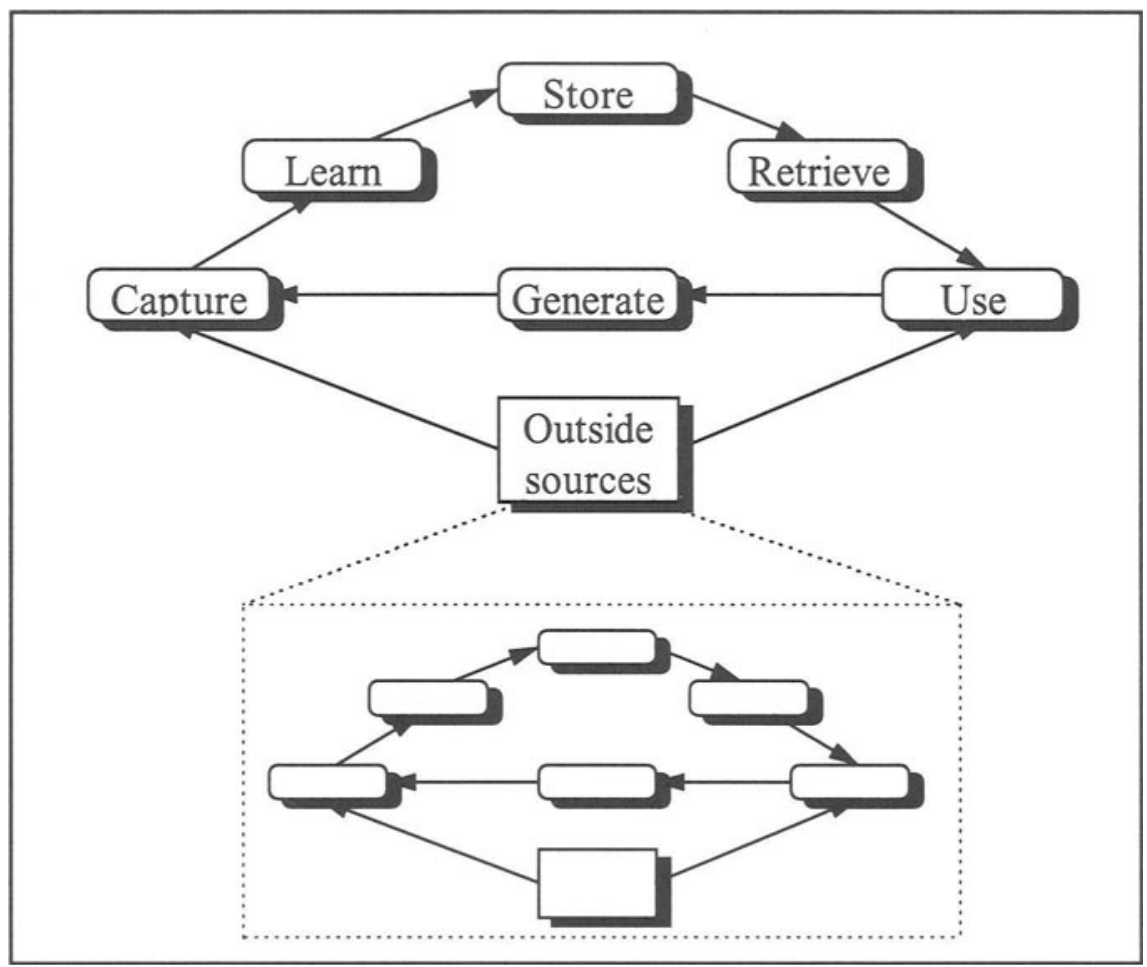

Figure 1. The knowledge life-cycle

Knowledge is generated throughout the design process, and in large quantities, by everyone involved. Capturing this knowledge after the event requires considerable additional effort and results in a retrospective account. Depending on the frequency of capturing, this may result in loss of important knowledge. Capturing knowledge therefore needs to be done in real time, that is as and when it is being generated and by the people who are generating it. Knowledge engineers can be involved at the start, to build the initial knowledge base, but subsequently their role should be no more than that of webmasters.

Capturing knowledge in real time has the advantage that knowledge can be made available soon after its creation. This improves the communication within a design team by having the latest information available on which to base decisions, in particular if these decisions require trade-offs between different aspects of the product.

The consequence of relying on a busy user to capture the knowledge means that it is unlikely that the generated knowledge will be abstracted or that a conscious effort to identify and extract reusable knowledge fragments 
will take place, as suggested in [Duffy et al 1995]. Instead, the knowledgecycle will have to be based on a basic learning process. This learning includes comparing the newly-captured knowledge with the stored knowledge and deciding on the required updates. Techniques such as machine learning will have to be used to derive meta-knowledge and generic knowledge from the captured and stored knowledge. It has been suggested that to make knowledge useful, some abstraction and transformation is needed [Mantyla 1996]. We think that the knowledge as it is generated and captured by the user, supported by automated indexing based on the context in which the knowledge is generated, can make this knowledge useful for reuse. Any abstraction may enhance the usefulness of the knowledge, but is not essential.

Ideally a design support system is only populated with knowledge that might be retrieved, rather than that what is known. There is little use in capturing the knowledge every designer is familiar with, unless this knowledge is stored to automate certain design tasks. We are interested in the knowledge that differentiates experts from novices; the knowledge and experience generated during design projects. As we described earlier, most of this knowledge currently comes from memory. The knowledge captured in memory is context dependent, is based on personal beliefs and opinions, and is often heuristic in nature. The main challenge is to capture this knowledge along with its context.

New structures are needed to store the knowledge as current product models are unsuitable. A system has been developed that addresses the knowledge life-cycle with the aim of capturing a greater proportion of what is generated during a design process, the context in which it is generated, as well as the rationale and other process-related knowledge. The next section gives an outline of the structure of the system. A discussion of how the system addresses the various stages in the knowledge life-cycle can be found in section 5 .

\section{A PROCESS-BASED SUPPORT SYSTEM}

The proposed system addresses the knowledge life-cycle in order to help improve the design process. Its main focus is on: capturing design data ${ }^{1}$ resulting from design activities throughout the design process; and assisting

${ }^{1}$ The use of the term "data" rather than knowledge in this context is based on the definition that what is generated is data until linked to other contextual data and processed in some way, that is until it obtains meaning. This implies that design data contains knowledge for the experienced user of that data. 
in the creation of this data. Design data contains the knowledge that is used and generated in a design project. As it is not possible to obtain a complete set of design data, it is important to ensure that one can represent and reason with what is captured, i.e. what one can expect the designer to make explicit within the constraints of an industrial context [Blessing 1996].

Design data includes:

- product data describing the product covering: all stages of development, including the requirements specification, functional diagrams, sketches, drawings, calculations, graphs, etc; the whole product-life cycle, including production plans, user manuals, maintenance instructions, etc; and all the alternatives that were considered at every stage of the process.

- process data including the rationale behind product data such as arguments and decisions related to the various stages of the product and the various alternatives that were considered; and the information to support the arguments, along with the various aspects of the business involved.

- process administration data including the planned and actually applied resources (who did what, when and how).

In particular, much process data is lacking in current project documentation, and so are descriptions of the various alternatives and the reasons why some of these were rejected.

Capturing design data is the most direct way of capturing knowledge and hence making it available. In an earlier paper [Blessing 1996] it was argued that:

- it would be ideal to capture all design data;

- only a small fraction is currently being captured;

- we cannot capture all data;

- we can capture more;

- a low level of indexing detail can be sufficient;

- capturing and indexing should be in real time;

- capturing and indexing should be based on the process.

\subsection{The PROSUS concept}

PROSUS, a PROcess-based SUpport System, is a workbench that aims to improve the design process by using a process model as its core to capture the data resulting from design activities, and to support the creation of these data throughout the process [Blessing 1994]. The process model:

- provides process-related guidance to the designer or design team, but allows different approaches;

- provides a structure for the design team to document and retrieve design data in all stages of the design process; 
- provides the basis for the system to determine the context in which the data were generated and use this to automatically index the data;

- enables the system to suggest relevant knowledge, methods, tools, design histories throughout the design process because these can be linked to steps in the design process.

PROSUS enables a shift in the task distribution and different levels of implementation to adapt to developments in computer technology, to new software for particular design activities, and to specific company characteristics.

PROSUS has three levels:

- primary level containing the basic building block of PROSUS, the Design Matrix, which is the main working area for the design team based on the process model;

- control level to determine the strategy, that is the sequence of steps in the process model or matrix;

- support level to determine the best support (method, tools, history) to execute a step.

This paper concentrates on the primary level, the core of which has been implemented. For more details about the other levels see [Blessing 1994].

\subsection{The core of PROSUS: the design matrix}

The basic building block of PROSUS is the design matrix. This matrix is based on a combination and extension of two complementary models: Methodical Design, a problem-oriented approach to design [Pahl and Beitz 1996, van den Kroonenberg 1978]; and IBIS, an Issue-Based Information System based on the approach to design proposed by Rittel [Kunz and Rittel 1970, McCall 1986].

The design matrix represents the design process as a structured set of issues and activities and is the interface between the user and the system. Figure 2 shows a simplified design matrix. For reasons of clarity, the various issues following "Concept", including assembly, installation, material, geometry, etc, have been grouped under the heading "Detail design".

Each cell can be envisaged as an area in which designers can document the results, both final and intermediate, of executing that particular step. Specific tools are not available for every step in the design process (a cell in the matrix), particularly during the early stages of the design process. For this reason, a default text tool and graphics sketching tool are linked to every cell. 
The rows of the design matrix cover the different issues that need to addressed during the design process ${ }^{2}$. The list of issues can be adapted to suit the approach of a specific company. The columns of the design matrix relate to the activities of the deliberation process needed to resolve an issue.

The left-hand column contains the relevant issues. Each issue can be addressed in three steps: Generate, Evaluate and Select. A Generate step results in proposals. This column may also contain data such as calculations or references to knowledge used to develop a proposal. Evaluation is the comparison of each proposal with the requirements and results in one or more candidate solutions, arguments and decisions. Arguments give the design rationale supporting or opposing a proposal. Arguments are the basis for taking decisions but can also lead to new issues or proposals. Decisions describe the status of a proposal. In the case of more than one candidate solution, a selection has to be made to decide upon the solution to pursue further by comparing the candidates. This activity results in a decision to select one or more solutions together with the arguments for this selection. Evaluation focuses to a large extent on the demands in the requirements list; selection focuses more on the wishes, and involves making trade-offs. As a consequence, different methods and tools are used for each of these activities.

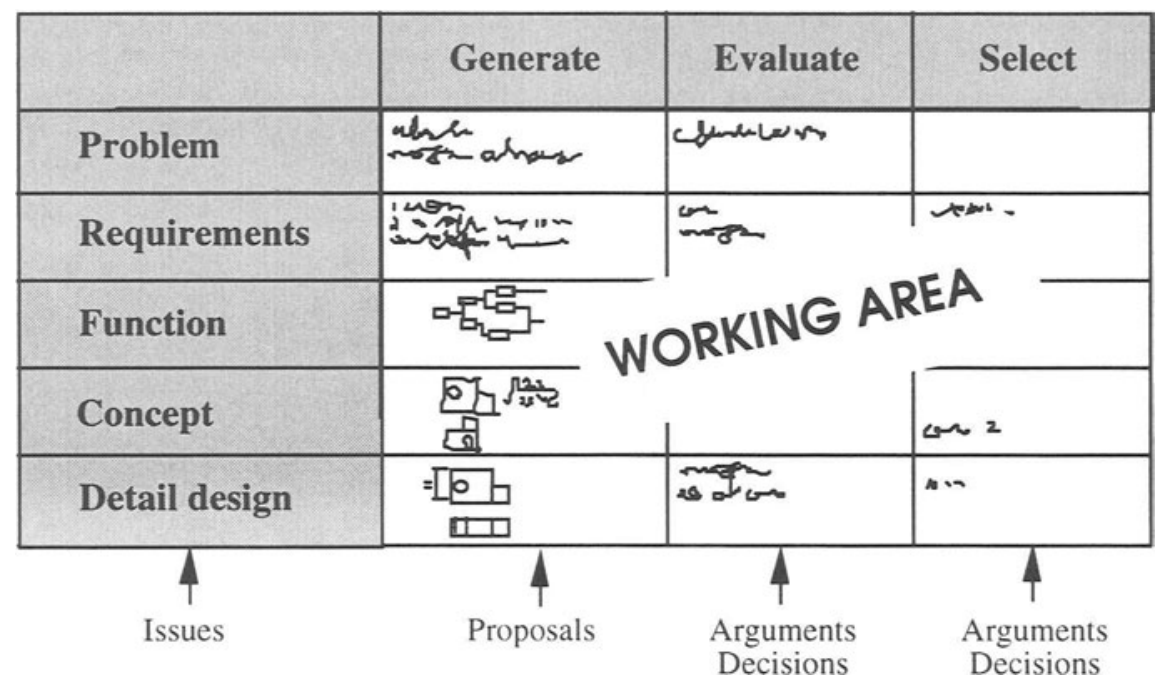

Figure 2. A simplified design matrix

${ }^{2}$ The word "issue" is often used to refer to a (potential) problem that needs attention, such as "reduce noise". In this context, problems such as "reduce noise" are considered to be aspects, probably formulated as requirements that are dealt with during evaluation or selection. 
The process model repeats itself in a slightly modified form for every assembly and component in the product. To support indexing and retrieval of the documented data, a separate matrix is used for each assembly (including the overall product) and component. Every design project therefore consists of a set of matrices, all linked together. The product and assembly matrices are identical. They only differ from the component matrices in the detail design issues they address. The matrix for standard components differs also in the first few issues.

\subsection{Capturing design data}

Data can be entered into the design matrix in any order; no sequence of addressing the cells is prescribed, nor do all cells have to be filled in. The cell in which the data is entered provides the context for the system to "understand" what the data is about and attach the relevant indices. These indices include the step in the process (the cell index), the user, the date and the time.

All data are captured in units called events. Every event is uniquely numbered within a project. An event relates to a specific combination of product element, issue, activity, method, tool, person and time. In terms of the matrix, this means that a move to another cell will start a new event, as does the use of a new method or tool, or another designer beginning to work on the project. In addition, designers can decide to start a new event, for example to generate a new alternative. Events can "live" in parallel.

When designing it is often not possible to know whether a product element will be an assembly or a component. The designer can choose to postpone this decision and start working on a matrix containing only the first four issues, which are identical for every matrix.

The system captures the various versions of the proposals that are generated to maintain a track record. In addition the system actively supports the generation and capture of alternatives.

Once completed, a design matrix contains a description of the design process for a specific product element. The description is structured around the rationale applied and may not be chronological. It is considered more important to see, for example, the various alternatives that were generated for a particular component clustered together, and the order in which they were generated, than to know exactly when they were generated. Each matrix structures the (intermediate) results of the process independently of the sequence in which they were generated, but, as each piece of data is automatically time-stamped, the chronological order can be retrieved when required. 


\subsection{Supporting the generation of design data}

A design matrix is more than a structured and passive notebook. The use of a process model allows PROSUS to suggest relevant knowledge, methods, tools and design histories, as these aids can be linked to specific activities, that is to one or more cells. When the designer is generating requirements, for example, the system is aware of this because it can detect the cell in which the designer is working. As a consequence the system can suggest to the user checklists and specification building tools, or the requirements list set up for the assembly one level up in the product hierarchy. The links between matrix cells and tools are currently structured as a direct links though the intention is to make them agent driven.

\subsection{Initial evaluation}

An initial evaluation of the use of the matrices in a design process focused on the applicability and usefulness of the design matrix concept [Blessing 1994, 1996]. The evaluation involved experienced designers and was based on a comparison of the design processes of two groups of designers: one working with the design matrices and one working without them. The findings were promising and justified further development. Since then a prototype has been implemented [Ball et al 1998] and is currently being evaluated.

\section{PROSUS AND THE KNOWLEDGE LIFE-CYCLE}

\subsection{Capture}

The use of the design matrix provides a way of capturing and indexing based on the context in which the data were generated. The context is defined by: the project; the product element and its relation to the rest of the product; the issue; the activity; the time; and the date. In addition, the author and the means used are captured. The event number (see 4.3) relates each data element to data captured in other events. This context is captured together with the data itself, which is only possible because the system is based on a process model.

PROSUS thus allows the capture of knowledge and its context as and when it is generated, and makes it immediately available (read-only) to other project members. It captures the dynamic and opportunistic nature of the design process, which is necessary to understand the decisions taken and the 
data that is used and generated. Obviously this requires the collaboration of the users, but the hope is that using PROSUS will encourage designers to document more of their processes. The initial evaluation of the matrix concept provided support for this hope.

\section{$5.2 \quad$ Learn}

Currently all that is captured is stored directly without any checks. The database is up-to-date because it contains the latest data. The users do consistency checks. The availability of all data, including the context, makes this task easier. Immediate availability, including the results of all the steps in the process, whether erroneous or not, are the primary drivers behind PROSUS.

It is assumed that it is better to have the various opinions stored next to each other with context, author and date, than to try to find the best opinion, even if this exists. This allows future users to determine what is relevant in their own situation. The fact that negative experiences are also captured is very important, as these constitute a large part of the lessons learnt. Nevertheless, more attention needs to be paid to the aspect of updating and real learning from the captured data as discussed in section 3 .

\section{$5.3 \quad$ Store}

Data elements are indexed and stored as and when they are generated. Retrospective capturing and indexing is not considered to be a feasible alternative given the enormous time pressures in industry and the potentially biased nature of recollecting events and decisions. The indexing system is based on what can be expected from the designers while working on developing a product, and on what indices could be captured automatically.

To enable the variety of captured data to be stored, a product model has been developed in which product as well as process data can be stored [Ball 1998].

\subsection{Retrieve}

PROSUS is based on the assumption that the whole knowledge life-cycle is essentially in the hands of the users, that is the design teams. This led to the decision to use the same indices for capturing and retrieving, and base these on the design process as well as the product. Typical search queries might be: show all concept alternatives that were generated for assembly $\mathrm{X}$ in project $\mathrm{Y}$; provide the reasons why a particular material was chosen; 
present the use and safety considerations of a particular component; and indicate the complete set of requirements used in a particular project.

The concept of events allows the replay of the history of an individual's activities; the activities related to a particular project or to a particular product element within a project; and also the events related to an earlier retrieved event. This linking of activities provides some understanding of the rationale in so far as this is not captured.

It would be useful if knowledge could be derived from what is captured and stored to support the retrieval of relevant data in projects with which the designer is unfamiliar. This is likely to require transformation and abstraction. Care should be taken that the context and any existing differences in opinion remain available, or at least accessible.

\subsection{Use}

Using PROSUS as a workbench would allow direct access to available methods, tools, knowledge and design histories. This would:

- encourage the consideration of knowledge generated in previous projects;

- support the use of up-to-date project related data;

- increase documentation of a project (e.g. for liability cases or reuse);

- encourage a more systematic approach to the process (project management);

- support teamwork by providing a communication aid.

In addition it could increase familiarity with and use of the various existing design tools in industry.

\subsection{Generation}

The use of PROSUS, and the supporting tools it suggests to the designer, supports the generation of new data, and allows this to be captured. Part of the newly created data will immediately be captured if a computer tool is used. However, most tools do not normally produce intermediate results and process-related data. Accessing the tools via PROSUS allows the system to capture at least some of the intermediate results and process-related data.

\section{CONCLUSIONS}

A mismatch has been observed between the information sources available to designers and the information sources accessed by designers. Possible reasons for this mismatch are lack of accessibility, availability and 
trustworthiness. Current support for product development does not appear to deal with this issue, because it only addresses part of the knowledge lifecycle A knowledge life-cycle was introduced that emphasises the generation of knowledge as the consequence of using knowledge.

The proposed design support system, PROSUS, was not developed to explicitly address the knowledge life-cycle. However, the concept is suitable to form the basis for knowledge life-cycle support because it is a way of indicating context, which is the basis of understanding. The system needs further development, in particular the issue of updating and extending the knowledge base by learning from the captured and stored data needs addressing.

\section{ACKNOWLEDGEMENTS}

The development of PROSUS has been undertaken in the Cambridge Engineering Design Centre supported by a grant from the Engineering and Physical Sciences Research Council (EPSRC). Further development and testing of PROSUS is one of the tasks of the new British Aerospace/RollsRoyce University Technology Partnership (UTP) for Design. This is a partnership between the two companies and the Universities of Cambridge, Sheffield and Southampton. The focus of the Cambridge UTP is on research into engineering knowledge management throughout the product life-cycle, the results of which will influence the future direction of the PROSUS system.

\section{REFERENCES}

Altschuller, G.S. (1984) Erfinden: Wege zur Lösung technischer Probleme, VEB Verlag Technik, Berlin.

Ball N.R., Matthews P.C. and Wallace K.M.(1998) Managing Conceptual Design Objects: An Alternative to Geometry, Proceedings AID98 (Eds. Gero, J. and Sudweeks, F.), Lisbon, 67-86, Kluwer, Dordrecht.

Blessing, L.T.M. (1994) A Process-Based Approach to Computer Supported Engineering Design, PhD Thesis, University of Twente, The Netherlands, published Cambridge.

Blessing, L.T.M. (1996) Design Process Capture and Support, Proceedings of the 2nd Workshop on Product Structuring (Eds. Tichem, M. et al), Delft, 109-121.

Carlton, C.(1998) The Retrieval of Mechanical Design Information, PhD Thesis, University of Cambridge.

Clausing, D. (1994) Total Quality Development, ASME Press, New York.

Court, A. (1995) The Modelling and Classification of Information for Engineering Designers, PhD Thesis, University of Bath. 
Duffy, S.M., Duffy, A.H.B. and MacCallum, K.J. (1995) A Design Reuse Model, Proceedings of ICED 95, Prague, 490-495, Heurista, Zurich.

Ehrlenspiel, K. (1997) Knowledge Explosion and its Consequences, Proceedings of ICED 97, Tampere, Vol. 2, 477-484, Heurista, Zurich.

Frankenberger, E. and Badke-Schaub, P. (1998) Integration of Group, Individual and External Influences in the Design Process, In Designers: The Key to Successful Product Development (Eds. Frankenberger, E., Badke-Schaub, P. and Birkhofer H.), 149-164, Springer-Verlag, Berlin.

Fricke, G. (1993) Konstruieren als flexibler Problemlöseprozeß: empirische Untersuchung über erfolgreiche Strategien und methodische Vorgehensweisen beim Konstruieren, Dissertation, University of Darmstadt, VDI Fortschrittberichte 1.227,VDI Düsseldorf.

Hales, C. (1987) Analysis of the Engineering Design Process in an Industrial Context, PhD Thesis, University of Cambridge, Gants Hill Publications, Hampshire.

Hubka, V. and Eder, W.E. (1988) Theory of Technical Systems, Springer-Verlag, Berlin.

Klaubert, H.L. (1998) Tiny Design: A Study of the Design of Microelectromechanical Systems, PhD Thesis, University of Cambridge University.

Kroonenberg, H.H. van den (1978) Methodisch Ontwerpen, Diktaat, University of Twente, Enschede, The Netherlands.

Kuffner, T. and Ullman, D.G. (1991) The Information Requests of Mechanical Design Engineering, Design Studies, Vol. 12, No. 1, 42-51.

Kunz, W. and Rittel, H. (1970) Issues as Elements of Information Systems, Center for Planning and Development Research, University of California, Berkely.

Mantyla, M. (1995) Knowledge-Intensive CAD: Introduction and a Research Agenda, Proceedings of IFIP WG 5.2 (Eds. Tomiyama, T., Mantyla, M. and Finger, S.), Finland, Chapman \& Hall, 3-12.

Marsh, J.R. (1997) The Capture and Utilisation of Experience in Engineering Design, PhD Thesis, University of Cambridge.

McCall, R.J. (1986) Issue-serve Systems: A Descriptive Theory of Design, Design Methods and Theories, Vol. 20, No. 3, 443-458.

Lera, S.G., Cooper, I. and. Powell J.A. (1984) Information and Designers, Design Studies, Vol. 5, No. 2, 113-120.

Pahl, G. and Beitz, W. (1996) Engineering Design, 3rd Edition, Springer-Verlag, London.

Pugh, S. (1991) Total Design, Addison-Wesley, Wokingham, UK. 